\title{
ANÁLISE DO ENSINO DE CIÊNCIAS NAS ESCOLAS DA REDE PÚBLICA DE
} SANTA CRUZ/RN

\section{Josiel Moreira da Silva}

Ciências e Educação Matemática, IFRN, Santa Cruz, Bolsista do Programa de Iniciação Científica, Aluno do Curso de Licenciatura em Física. E-mail: josiel1nico@ hotmailcom.

\section{Lauricéia Lays Santos Valença}

Ciências e Educação Matemática, IFRN, Santa Cruz, Bolsista do Programa de Iniciação Científica, Aluno do Curso de Licenciatura em Física. E-mail: lauriceia_lays@ @otmail.com.

\section{Falconiere Leone Bezerra de Oliveira}

Ciências e Educação Matemática, IFRN, Santa Cruz, Programa de Iniciação científica, Aluno do curso de Especialização em Geografia do Semi-árido. E-mail: falconi_rn@yahoo.com.br.

\section{Leandro Silva Costa}

Ciências e Educação Matemática, IFRN, Santa Cruz, Programa de Iniciação Científica, Professor de Biologia. E-mail: Leandro.costa@ifrn.edu.br.

\section{RESUMO}

O quadro atual do ensino de Ciências no Brasil, principalmente nas escolas públicas, é extremamente preocupante, seja pela falta de recursos nas escolas, inexistência de laboratórios e/ou equipamentos adequados e ainda pela existência de docentes sem formação específica e com métodos de ensino ultrapassados. Baseado nesses dados, nós desenvolvemos um trabalho cujo objetivo foi realizar um diagnóstico do ensino de ciências nas escolas públicas do município de Santa Cruz/RN. A pesquisa foi executada através de entrevistas estruturadas com auxilio de questionários destinados a 20 professores de 7 escolas públicas, tendo como eixos temáticos o perfil socioeconômico e a formação profissional do professor, bem como a análise da estrutura física das escolas. Pode-se constatar que $89 \%$ dos professores investigados recebem no máximo três salários mínimos, enquanto apenas $11 \%$ recebem de 4 a 8 salários. Com relação à formação acadêmica, apenas $53 \%$ dos professores entrevistados apresentam formação na área, enquanto $47 \%$ não possuem formação específica ou não concluíram o curso de graduação. No que diz respeito à infra-estrutura, apesar de $66 \%$ das escolas possuírem laboratório de informática, nenhuma dispõe de um laboratório para o desenvolvimento de aulas práticas de ciências. Esses resultados nos mostram a urgente necessidade de se desenvolver estratégias que busquem aprimorar o ensino de ciências na região, o que aparece como uma oportunidade do IFRN-Santa Cruz se inserir neste contexto, através principalmente da oferta de cursos de formação docente continuada.

PALAVRAS-CHAVE: formação profissional, perfil do educador, infra-estrutura escolar.

\section{ANALYSIS OF SCIENCE TEACHING IN PUBLIC SCHOOLS OF SANTA CRUZ, RN}

\begin{abstract}
The current situation of science teaching in Brazil, mainly in public schools is extremely disturbing, by absence of resources in schools, deficiency of laboratories and / or appropriate equipment and existence of teachers without specific training and teaching methods obsolete. Based on these data, we developed a project whose objectives were to diagnosticate the
\end{abstract}


science education in public schools in Santa Cruz, RN. The research was performed through structured interviews with the assistance of questionnaires to 20 teachers from seven public schools, based in the socioeconomic profile of teacher training, as well as analysis of the physical structure of schools. It can be seen that $89 \%$ of teachers surveyed receive up to three wages, while only $11 \%$ receive $4-8$ wages. With respect to academic training, only $53 \%$ of the interviewed teachers have training in the area, while $47 \%$ have no formal training or have not completed their undergraduate courses. With regard to infrastructure, although $66 \%$ of schools have computer labs, none offers a laboratory for the development of practical in science. These results show the urgent need to develop strategies to improve science education in the region, which appears as an opportunity to IFRN-Santa Cruz, mainly by offering teacher training courses.

KEY-WORDS: Teacher training courses; Teacher profile; Infrastructure. 


\section{ANÁLISE DO ENSINO DE CIÊNCIAS NAS ESCOLAS DA REDE PÚBLICA DE} SANTA CRUZ/RN

\section{INTRODUÇÃO}

O ensino de Ciências no Brasil, principalmente no Ensino Fundamental, tem enfrentado dificuldades no que se refere ao desenvolvimento da prática pedagógica dos professores dessa área e isso decorre do pouco tempo em que o Ensino de Ciências foi incorporado no sistema educacional brasileiro (PIMENTA, 1999).

De acordo com os Parâmetros Curriculares Nacionais (1997), o ensino de ciências tornou-se obrigatório com a promulgação da Lei de Diretrizes e Bases da Educação de 1961, período em que o ensino tradicional predominava e o conhecimento científico tinha pouca relevância. O quadro atual do ensino de Ciências no Brasil, principalmente nas escolas públicas, é extremamente preocupante, seja pela falta de recursos nas escolas, inexistência de laboratórios e/ou equipamentos adequados e ainda pela existência de docentes sem formação específica e com métodos de ensino ultrapassados (ACADEMIA BRASILEIRA DE CIÊNCIAS, 2007). Dessa forma, a organização do ensino de Ciências vem se acentuar em inúmeras propostas para a sua modificação onde poderá proporcionar uma assimilação ativa dos conhecimentos para os alunos. Tal assimilação proporcionará uma melhor condição na formação do espírito cientifico discente, tendo com aparato as condições histórico-culturais da sociedade.

O ensino de ciências sempre foi subjugado apenas a meras transmissões de conteúdos em grande escala, onde a qualidade do curso era baseada na quantidade de conteúdos ministrados, isso até os anos 60. Na década de 70, com a crise econômica, decorrente de uma quebra com o modelo desenvolvimentista deflagrado após a segunda guerra mundial surgiu no ensino de ciências um movimento pedagógico que ficou conhecido como "Ciências, Tecnologia e Sociedade" (CTS) que tomou corpo nos anos 80 e respalda até hoje. Nos anos 80 o modelo de ensino de Ciências era baseado basicamente na concepção da diferentes correntes construtivistas, que hoje é bem aceito por muitos pesquisadores, contudo os Parâmetros Curriculares Nacionais (PCNs) remete que:

\footnotetext{
Esse modelo tem merecido críticas que apontam a necessidade de reorientar as investigações para além das pré-concepções dos alunos. Não leva em conta que a construção de conhecimento científico tem exigências relativas a valores humanos, à construção de uma visão de Ciência e suas relações com a Tecnologia e a Sociedade e ao papel dos métodos das diferentes ciências. (PCNs, 1997, p. 23)
}

Preocupados com a situação em que se encontra o ensino de ciências, desenvolvemos uma pesquisa com o objetivo de realizar um diagnóstico do ensino de ciências nas escolas públicas do município de Santa Cruz/RN, mais precisamente no Ensino Fundamenta II ( $6^{\circ}$ ao $9^{\circ}$ ano).

Para tomar consciência desse processo investigativo utilizamos como metodologia uma pesquisa de natureza qualitativa que foi executada através de entrevistas estruturadas com auxilio de questionários destinados a 20 professores de 7 escolas públicas tendo como eixos temáticos o perfil socioeconômico e a formação profissional do professor, bem como a análise da estrutura física das escolas.

Então, percebemos que a precariedade em que se encontra o ensino de ciências, decorre de vários fatores contribuintes tais como a falta de recursos nas escolas, inexistência de laboratórios e/ou equipamentos adequados e ainda pela existência de docentes sem formação específica e com métodos de ensino ultrapassados (LIMA \& VASCONCELOS, 2006; 
OLIVEIRA et al, 2009) o que contribui para que os professores da área se acomodem e não busquem se atualizar, para melhorar a sua prática pedagógica dentro da sala de aula.

\section{RESULTADOS E DISCUSSÕES}

Dos vinte questionários aplicados, um total de dezessete foram devidamente respondidos e devolvidos pelos entrevistados. Através das entrevistas podemos observar que a maioria dos professores encontram-se entre a faixa etária de 30 a 35 anos $(64,7 \%)$, enquanto $29,4 \%$ dos entrevistados possuem menos de 30 anos e apenas 5,9\% possuem idade acima dos 35 anos. Em relação ao sexo dos professores, existe predominância de mulheres $(82,4 \%)$, conforme mostra a tabela 1 .

Tabela 1: Sexo dos professores das escolas de rede pública de Santa Cruz - RN.

\begin{tabular}{|c|c|c|}
\hline \multirow{2}{*}{ SEXO } & População (n) & $\%$ \\
\hline \multirow{2}{*}{ Masculino } & 3 & 17,6 \\
\hline Feminino & 14 & 82,4 \\
\hline
\end{tabular}

Através da Figura 1, podemos constatar que a grande maioria dos professores investigados $(89 \%)$ recebe no máximo três salários mínimos, enquanto apenas $11 \%$ recebem de quatro a oito salários. O baixo nível salarial observado aqui corrobora com dados de pesquisas feitas com professores da rede pública do Nordeste (LIMA \& CAVALCANTI, 2006; OLIVEIRA et al, 2009). Entretanto, é interessante destacar que além da péssima remuneração, ao contrário do que se observa nessas capitais, nenhum professor de Santa Cruz declarou receber mais que oito salários, mostrando que praticamente não existe a oportunidade destes virem a ser bem sucedidos profissionalmente, o que segundo BAZZO (2000), em longo prazo pode se tornar um fator desestimulante para os alunos dos cursos de licenciatura. 


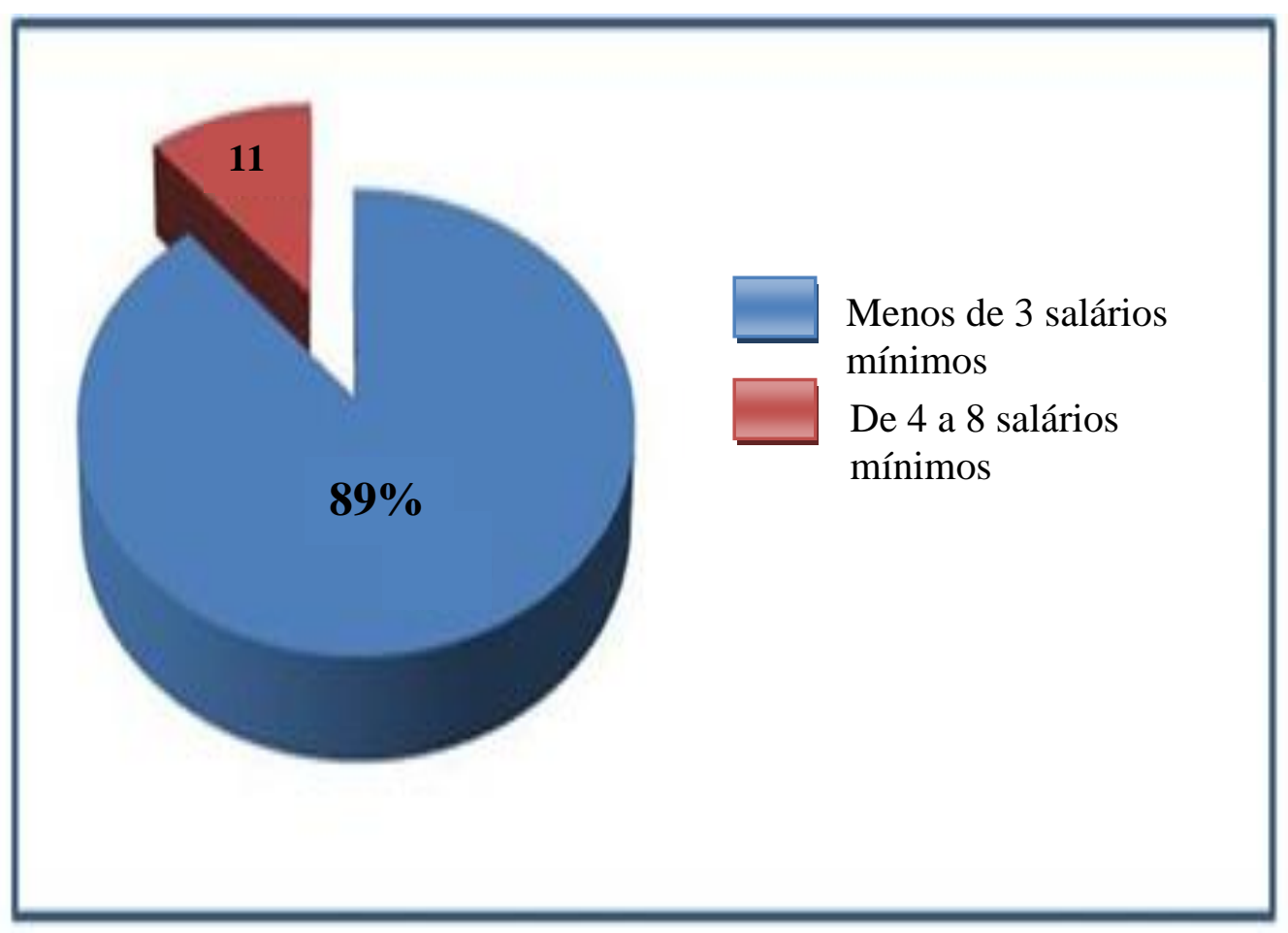

Figura 1 - Faixa Salarial dos professores de Ciências da rede pública do município de Santa Cruz -RN.

A análise dos dados referentes à formação acadêmica dos professores entrevistados é mostrada na figura 2. Pode-se observar que $47 \%$ dos professores não possuem formação específica ou não concluíram o curso de graduação, enquanto apenas 53\% apresentam formação acadêmica na área. Esses dados se tornam ainda mais preocupante quando se leva em consideração que dentre os professores com formação na área, apenas 33\% destes fizeram cursos de atualização profissional na área de ensino de ciências nos últimos cinco anos. 


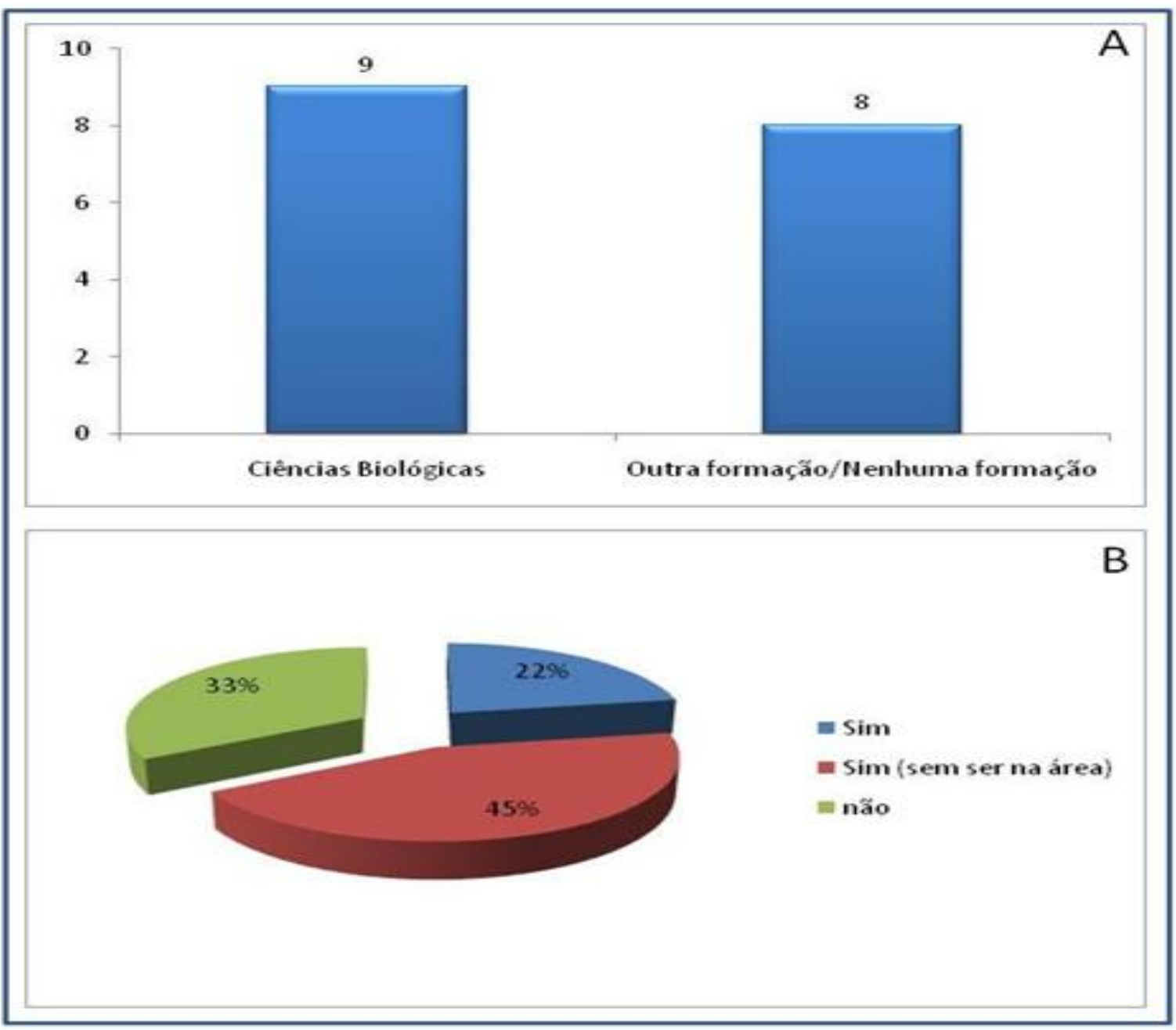

Figura 2 - Formação profissional dos professores de Ciências da rede pública do município de Santa Cruz -RN. a) Formação acadêmica; b) Atualização profissional nos últimos cinco anos dos professores com curso superior na área de Ciências.

O profissional que atua no ensino de ciências está incumbido de trabalhar conteúdos específicos que abrange várias áreas das ciências, tendo que orientar-se pelos eixos norteadores, como também trabalhar em seu currículo com diversos temas transversais, portanto, a falta de formação profissional direcionada para as ciências contribui com o surgimento de uma série de dificuldades metodológicas nos educadores, que passam a fazer uso de um modelo didático tradicional, caracterizado por concepções de ensino como transmissão/transferência de conhecimentos, aprendizagem receptiva e por um conhecimento absolutista e racionalista (KRASILCHIK, 2004).

No que diz respeito à infra-estrutura, apesar de $66 \%$ das escolas possuírem laboratório de informática, nenhuma dispõe de um laboratório para o desenvolvimento de aulas práticas de ciências. É inquestionável a importância das atividades experimentais no processo ensinoaprendizagem promovendo melhores condições de aprendizagem aos alunos, bem como de elaboração do conhecimento e desenvolvimento (CARVALHO et al, 2010). Apesar da inexistência de laboratórios nas escolas de Santa Cruz, os professores afirmam buscar minimizar esta deficiência através de atividades práticas alternativas feitas em sala de aula, o 
que por sua vez proporciona resultados semelhantes àqueles vistos em aulas práticas desenvolvidas dentro do laboratório de ciências.

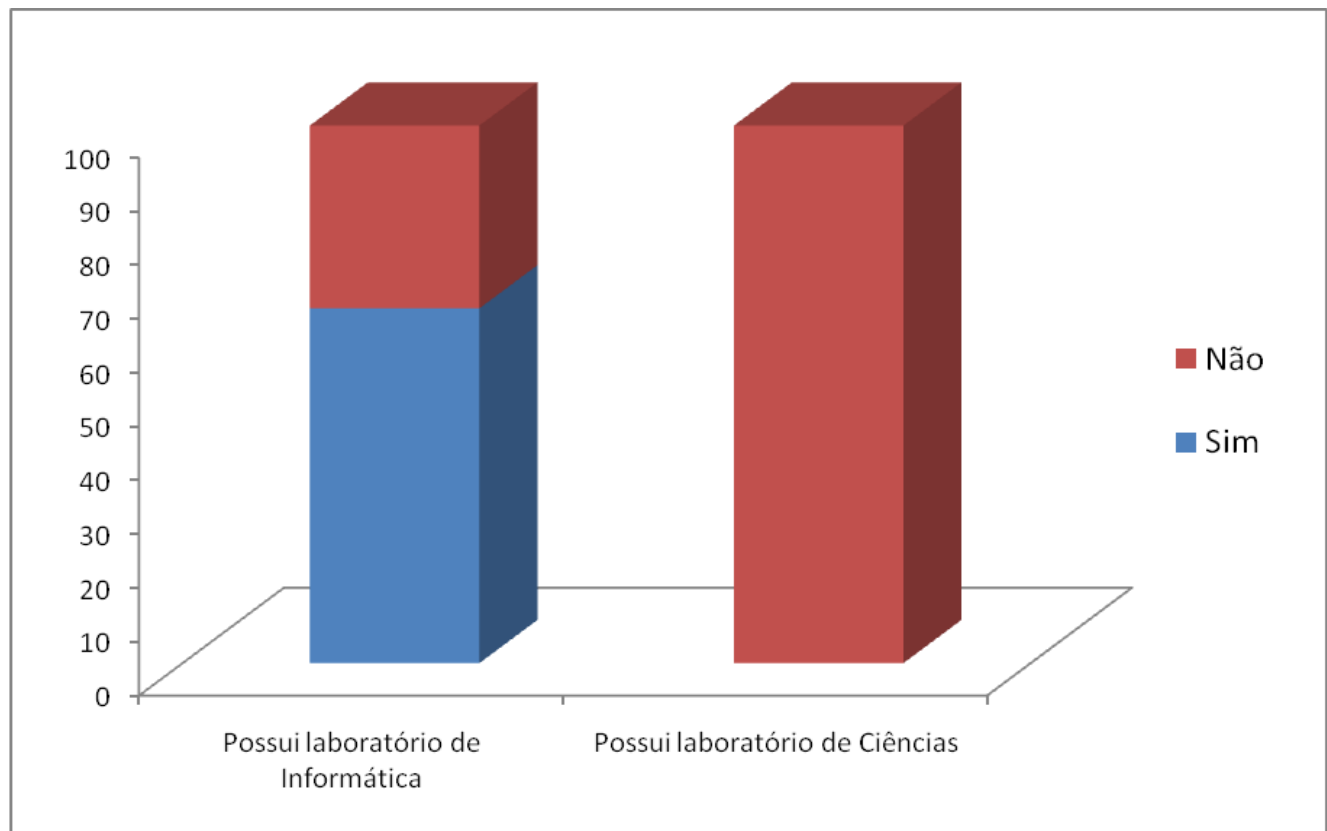

Figura 3 - Existência de laboratórios nas escolas da rede pública do município de Santa Cruz -RN.

\section{CONCLUSÕES}

Nossa pesquisa teve como ponto de partida a necessidade de desenvolver um diagnóstico do ensino de ciências no município de Santa Cruz/RN. Os resultados apresentados nesta pesquisa mostram a urgente necessidade de se desenvolver estratégias que busquem aprimorar o ensino de ciências na região. Acreditamos que somente um processo em médio prazo de valoração do professor, que permita o investimento em pesquisa educacional, poderá gerar aprendizagem transformadora, o que aparece como uma oportunidade do IFRN-Santa Cruz se inserir neste contexto e atuar por meio da oferta de cursos de atualização profissional dos professores, principalmente de cursos de formação docente continuada, desenvolvendo, junto com os professores, métodos dinâmicos de ensino o que tornará as aulas mais atrativas e práticas com o uso de materiais concretos. Tudo isso contribuirá para o melhoramento das práticas pedagógicas dos professores e, por conseguinte, do ensino de ciências da região.

\section{AGRADECIMENTOS}

Agradecemos aos professores que se dispuseram a participar de nossa pesquisa, bem como aos diretores de escolas pelo apoio disponibilizado. O aluno Josiel Moreira da Silva é grato ao apoio financeiro fornecido pelo Programa de Iniciação Científica do IFRN.

\section{REFERÊNCIAS}

1. BAZZO, V. L. Para onde vão as licenciaturas?: a formação de professores e as políticas públicas. Educação, Santa Maria, RS, v. 25, n. 1, p. 53-65, 2000. 
2. BRASIL. Secretaria de Educação Fundamental. Parâmetros curriculares nacionais: ciências naturais. - Brasília: MEC/SEF, 1997.

3. CARVAlHO, U. L. R.; PEREIRA, D. D.; MACEDO, E.; SILVA, K.; CIBELI, M.; FOLENA, M. A Importância Das Aulas Práticas De Biologia No Ensino Médio. X.

4. Jornada De Ensino, Pesquisa E Extensão - Jepex. Universidade Federal Rural de Pernambuco, Recife. 2010.

5. KRASILCHIK, M. Prática de ensino de biologia. 4. ed. São Paulo: Ed. da USP, p.198, 2004

6. LIMA, K. E. C.; VASCONCELOS, S. D. Análise da metodologia de ensino de ciências nas escolas da rede municipal de Recife. Ensaio: aval. pol. públ. Educ., Rio de Janeiro, v.14, n.52, p. 397-412. 2006

7. OllVEIRA G. A.; DONATO C. R.; SANTOS M; DANTAS M. A. T. Principais fatores que motivam os professores de ensino de Ciências e/ou Biologia do município de Aracaju, Sergipe a lecionarem SCIENTIA PLENA VOL. 5, NUM. 3 2009.

8. PIMENTA, Selma G. Saberes pedagógicos e atividade docente. 2 ed. São Paulo: Cortez, 1999.

9. ACADEMIA BRASILEIRA DE CIÊNCIAS. Ensino de ciências e educação básica: propostas para um sistema em crise. Rio de Janeiro, 2007. 